\title{
PREPARAÇÃO DE UM CENTRO CIRÚRGICO DO NORDESTE DO BRASIL PARA CIRURGIAS DURANTE A PANDEMIA DA COVID-19
}

\author{
Preparation of a surgical center in Northeast Brazil for surgeries during the COVID-19 pandemic
}

Preparación de un centro quirúrgico de Noreste de Brasil para cirugías durante el pandemia de COVID-19

\author{
Eduardo Tavares Gomes ${ }^{1 *}$ (), Marcone César Tabosa Assunção² (D), Mayana Camila Barbosa Galvão ${ }^{3}$ (D), \\ Jacqueline Augusta do Nascimento Oliveira ${ }^{4}$ (1), Caline Sousa Braga Ferraz ${ }^{5}$ (D), Priscilla Glazielly dos Santos de Moraes ${ }^{6}$ (D), \\ Cinthia Regina Albuquerque de Souza ${ }^{7}$, Mauricia Figueiroa da Silva ${ }^{8}$ (B)
}

RESUMO: Objetivo: Relatar a experiência da preparação do centro cirúrgico de um hospital universitário brasileiro para o atendimento de cirurgias em pacientes suspeitos e confirmados de COVID-19. Método: Relato da experiência do centro cirúrgico de um hospital universitário do Nordeste do Brasil, em março e abril de 2020, para o atendimento durante a pandemia da COVID-19. Resultados: A implementação do protocolo de atendimento a pacientes suspeitos e confirmados de COVID-19 foi realizada por meio da sua elaboração por um time de profissionais norteados por diretrizes nacionais e internacionais, treinamentos e atualizações. Os ciclos de aprimoramento permitiram que o protocolo fosse aperfeiçoado ao longo dos dois meses relatados. Barreiras e facilitadores para o processo são discutidos e estratégias são elaboradas para adequações possíveis. Conclusão: Houve adequação de protocolos assistenciais relacionados à rotina de fluxo de pacientes e da assistência em sala operatória no intuito de melhor atender os pacientes e reduzir os riscos de contaminação de outros pacientes e profissionais. Palavras-chave: Centros cirúrgicos. Salas de cirurgia. Enfermagem perioperatória. Infecções por coronavírus.

ABSTRACT: Objective: To report the experience of preparing the operating room of a Brazilian university hospital for surgeries for patients suspected and confirmed for COVID-19. Method: Experience report of the operating room of a university hospital in Northeast Brazil, conducted in March and April 2020, for care during the COVID-19 pandemic. Results: The implementation of the care protocol for suspected and confirmed cases of COVID-19 was carried out through its elaboration by a team of professionals based on national and international guidelines, training and updates. Improvement cycles allowed the protocol to be refined over the two months reported. Barriers and facilitators to the process are discussed and strategies are drawn up for possible adjustments. Conclusion: There was an adaptation of care protocols related to the routine of patient flow and care in the operating room to better serve patients and reduce the risk of contamination of other patients and professionals.

Keywords: Surgicenters. Operating rooms. Perioperative nursing. Coronavirus infections.

RESUMO: Objetivo: Informar la experiencia de la preparación del centro quirúrgico de un hospital universitario brasileño para la atención de cirugías en pacientes sospechosos y confirmados de COVID-19. Método: Informe de la experiencia del centro quirúrgico de un hospital universitario del Noreste del Brasil, en marzo y abril de 2020, para la atención durante la pandemia de COVID-19. Resultados: La implementación del protocolo de la atención

'Doutorando em Ciências pelo Programa de Pós-graduação em Enfermagem em Saúde do Adulto da Escola de Enfermagem da Universidade de São Paulo. Enfermeiro assistencial da Unidade de Centro Cirúrgico do Hospital das Clínicas/Universidade Federal de Pernambuco (UFPE) - Recife (PE), Brasil.

2Doutorando em Gestão e Economia da Saúde pela UFPE - Recife (PE), Brasil.

${ }^{3}$ Mestre em Enfermagem pela Universidade Federal do Rio Grande do Norte. Enfermeira assistencial da Unidade de Centro Cirúrgico do Hospital das Clínicas/UFPE - Recife (PE), Brasil.

4Mestre em Ergonomia pela UFPE. Enfermeira assistencial da Unidade de Centro Cirúrgico do Hospital das Clínicas/UFPE - Recife (PE), Brasil.

${ }^{5}$ Especialista em Enfermagem em Saúde da Criança pelo Programa de Residência em Enfermagem do Instituto de Medicina Integral Professor Fernando Figueira (IMIP). Enfermeira assistencial da Unidade de Centro Cirúrgico do Hospital das Clínicas/UFPE - Recife (PE), Brasil.

¿Especialista em Enfermagem Cirúrgica pelo Programa de Residência em Enfermagem do IMIP. Enfermeira assistencial da Unidade de Centro Cirúrgico do Hospital das Clínicas/UFPE - Recife (PE), Brasil.

${ }^{7}$ Mestranda em Avaliação em Saúde pelo IMIP. Enfermeira assistencial da Unidade de Centro Cirúrgico do Hospital das Clínicas/UFPE - Recife (PE), Brasil.

${ }^{8}$ Enfermeira assistencial do Hospital das Clínicas da UFPE - Recife (PE), Brasil.

*Autor correspondente: edutgs@hotmail.com

Recebido: 27/06/2020 - Aprovado: 06/05/2021

https://doi.org/10.5327/Z1414-4425202100020008 
a los pacientes sospechosos y confirmados con COVID fue llevada a través de la elaboración por un equipo de profesionales guiados por las directrices nacionales e internacionales, entrenamientos y actualizaciones. Los ciclos de mejora permitieron mejorar el protocolo durante los dos meses informados. Las barreras y los facilitadores para el proceso se discuten, y las estrategias son elaboradas para los ajustes posibles. Conclusión: Se adecuaron protocolos asistenciales relacionados a la rutina del flujo de pacientes y de la asistencia en centro quirúrgico, con la intención de atender mejor a los pacientes y reducir los riesgos de la contaminación de otros pacientes y profesionales.

Palabras-clave: Centros quirúrgicos. Quirófanos. Enfermería perioperatoria. Infecciones por coronavirus.

\section{INTRODUÇÃO}

A pandemia da COVID-19 (Coronavirus Disease, 2019) chegou ao Brasil, com transmissão comunitária, no fim do mês de março de $2020^{1}$. Em meio a muitas incertezas, os profissionais do centro cirúrgico (CC) aumentavam seus níveis de tensão diante da exposição ao novo vírus.

As salas operatórias $(\mathrm{SO})$ foram reconhecidas, desde o começo da pandemia, como locais com grande potencial de contaminação e disseminação do vírus². Os CC necessitavam preparar-se para a proteção dos seus profissionais ${ }^{3}$. De início, acreditava-se que a maior preocupação seriam os pacientes sintomáticos respiratórios, considerando-se que a confirmação dos casos demorava dias diante da escassez de exames na rede de saúde do Brasil nos meses de fevereiro e março. Contudo, com o início da transmissão comunitária no país e na localidade do hospital em estudo, a capital de um estado do Nordeste do Brasileiro, qualquer paciente e até mesmo os profissionais que trabalhavam juntos podiam ser fontes de contaminação. Nesse cenário, os alertas para racionamento de materiais e a possibilidade de escassez de equipamentos de proteção individual (EPI) também eram geradores de tensão $0^{1,46}$.

Além disso, muitos profissionais mantinham vínculos empregatícios com mais de um estabelecimento de saúde, aumentando sua exposição e favorecendo a circulação do vírus. Por mais que em um serviço não fossem realizadas cirurgias em pacientes suspeitos, os assintomáticos passaram a ser considerados potenciais fontes, assim como os profissionais do time cirúrgico.

Muitos departamentos cirúrgicos relataram como a pandemia tem impactado nas suas rotinas de agendamento de cirurgias, alterado suas filas e sua forma de priorização. Contudo, há significativa falta de discussões de como a pandemia influenciou as rotinas, dentro da SO, de manejo da paramentação, montagem, desmontagem e desparamentação $o^{7-12}$.

\section{OBJETIVO}

Relatar a experiência da preparação do CC de um hospital universitário para o atendimento de cirurgias em pacientes suspeitos e confirmados de COVID-19, na adequação de seus protocolos assistenciais relacionados à rotina de $\mathrm{SO}$.

\section{MÉTODO}

Trata-se de um relato de experiência ocorrido no CC de um hospital universitário localizado na região Nordeste do Brasil, em março e abril de 2020, para o atendimento na pandemia da COVID-19. O serviço conta com 10 SO de médio e grande porte para cirurgias de alta complexidade e mais quatro $\mathrm{SO}$ de pequeno porte para cirurgias ambulatoriais. Nesse hospital, o centro obstétrico é separado do CC. Além disso, há duas salas de recuperação pós-anestésica (SRPA), uma para cada bloco de SO. O processo de implementação das medidas seguiu a orientação do ciclo plan, do, check, act (PDCA).

O presente relato foi aprovado pelo Comitê de Ética em Pesquisa da Instituição.

\section{RESULTADO: RELATO DA EXPERIÊNCIA}

A unidade iniciou pela suspensão temporária de cirurgias ambulatoriais e a priorização das cirurgias de maior porte e de urgência, tendo em vista a crise de abastecimento de materiais e de EPI. Além disso, ao longo desses dois meses (março e abril de 2020), diversos profissionais foram afastados dos seus postos de trabalho por serem de grupos de risco ou por terem sido contaminados com o vírus, o que impediu o funcionamento do CC em toda sua plenitude.

Em seguida, um time formado pelos autores deste relato iniciou o processo de elaboração de um protocolo para a assistência aos pacientes contaminados com o coronavírus SARS-CoV-2. Profissionais do Serviço de Controle de Infecção Hospitalar (SCIH) e da Unidade de Gerência de Riscos Assistenciais (UGRA) prepararam orientações e fluxos para o hospital, que foram utilizados, particularmente os protocolos de higiene das mãos e uso de EPI. O uso de EPI tinha a inclusão de um equipamento que ainda não era 
utilizado nos CC do Brasil, a face shield (máscara facial), além da rotina de paramentação e desparamentação específica.

O time de profissionais, conduzido pela chefia do departamento de CC, iniciou o processo de construção de um protocolo próprio baseado em evidências. Após iniciado o processo e concluída a primeira versão do protocolo institucional, foram divulgadas diretrizes dos órgãos que orientam a prática em $\mathrm{SO}$ e que foram consultadas e seguidas na elaboração do documento. As diretrizes e orientações adotadas foram as da Sociedade Brasileira de Enfermeiros de Centro Cirúrgico, Recuperação Anestésica e Centro de Material e Esterilização (SOBECC), da Sociedade Brasileira de Anestesiologia (SBA), da Association of periOperative Registered Nurses (AORN), da Surgical Infection Society, do Centers for Disease Control and Prevention (CDC), entre as citadas em outras revisões sobre o tema ${ }^{13-18}$.

A Figura 1 apresenta, de forma esquemática, o processo como um ciclo PDCA.

A etapa de planejamento (plan - P) incluiu desde a consulta às fontes e orientações até a elaboração do protocolo. A etapa do fazer $($ do $-\mathrm{D})$ incluiu todos os treinamentos ministrados. Ao todo, participaram do treinamento 58 profissionais, em seus turnos de trabalho. A etapa de conferir o protocolo (check - C) ocorreu quando os primeiros pacientes começaram a ser atendidos, em abril de 2020. À medida que os atendimentos ocorriam e melhoras possíveis ao protocolo iam sendo aventadas, o time responsável avaliava e entrava em ação, fazendo ajustes ao protocolo e divulgando as atualizações entre as equipes envolvidas (act - A).

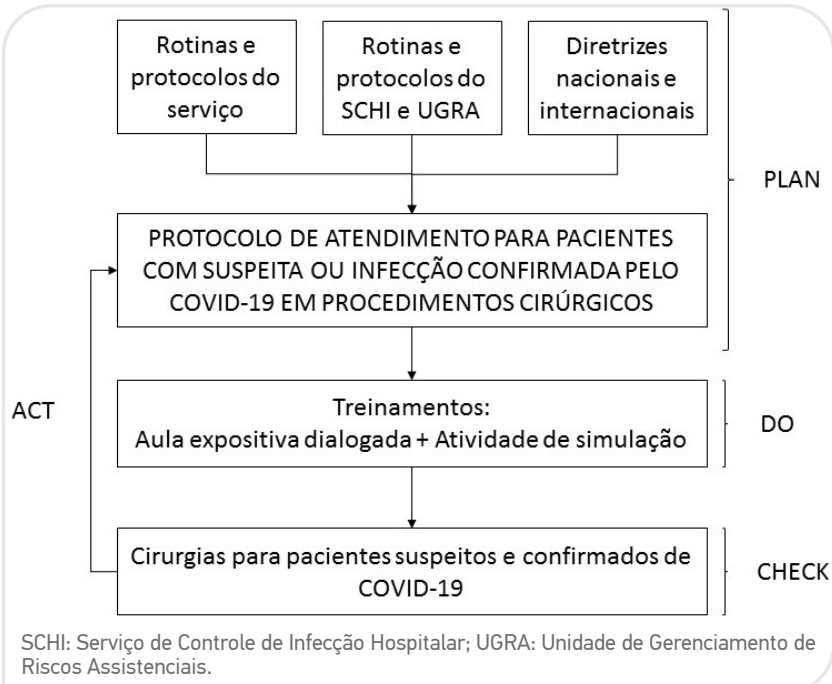

Figura 1. Implementação do protocolo de atendimento para pacientes com suspeita ou infecção confirmada pela COVID-19 em procedimentos cirúrgicos.
O protocolo teve duas versões principais em decorrência das mudanças também ocorridas nas diretrizes seguidas. Os treinamentos iniciaram às vésperas de se concluir a segunda versão. Elaborou-se um treinamento com uma apresentação expositiva dialogada, seguida de uma atividade prática de paramentação e desparamentação, depois uma simulação de montagem e desmontagem de SO de acordo com o protocolo. Os profissionais foram treinados no próprio setor e pelo próprio time que fez a elaboração teórica do protocolo e foi atingida a marca de $80 \%$ dos profissionais treinados no serviço, considerando-se os afastamentos no período. A etapa inicial do treinamento de paramentação e desparamentação também foi ofertada para profissionais de anestesiologia, para cirurgiões e médicos residentes e para profissionais do serviço de limpeza. O time das unidades de terapia intensiva (UTI) ofereceu os treinamentos de intubação orotraqueal e reanimação cardiopulmonar para todo o hospital, incluindo os profissionais do CC.

\section{Resumo das adaptações do protocolo para atendimento de suspeitos e confirmados de COVID-19 em sala operatória}

Para se alcançar sucesso em todas as etapas do protocolo, a comunicação é primordial. As equipes cirúrgicas devem comunicar com antecedência o CC sobre o status do paciente para que o protocolo seja iniciado.

Para uma cirurgia, são destinadas duas SO: uma na qual ocorre a paramentação antes da cirurgia e a desparamentação em seguida e outra na qual se realiza a cirurgia propriamente dita, após as adaptações na montagem da sala. As SO são identificadas na porta com placas de isolamento de contato, isolamento respiratório e identificação de COVID-19.

Algumas rotinas já eram realizadas em cirurgias infectadas ou para pacientes em isolamento de contato, como a retirada do máximo de material e mobiliário da SO. Além disso, até a indução anestésica já era realizado o desligamento do ar-condicionado para prevenção de hipotermia e, no protocolo, a prática permaneceu até após a intubação orotraqueal, procedimento gerador de aerossóis ${ }^{16,18-21}$.

A estação de trabalho da anestesia (work station) era preparada com filtro bacteriano para o circuito ventilatório, com a colocação de uma pinça de preensão para o tubo orotraqueal envolta em filme plástico ${ }^{16,18-21}$. A pinça deve ser utilizada sempre que ocorrer abertura do sistema fechado de ventilação da via área ${ }^{16,18-21}$. Também deve ser vedada a via proximal do tubo orotraqueal, permitindo apenas a passagem do bougie ou guia, sem a saída de aerossóis. 
O uso do filme plástico foi adotado em todo mobiliário, incluindo console de bisturi, bombas de infusão e aspiradores. Na desmontagem da SO, a rotina é desinfetar por cima do filme com solução de álcool a 70\%, retirar o filme e desinfetar novamente a superfície.

A permanência de pessoas na SO foi reduzida, excluindo-se, por exemplo, alunos de graduação. Os técnicos de enfermagem, circulantes de SO, são escalados para a cirurgia sempre aos pares: um para dentro da $\mathrm{SO}$ e outro para permanecer à porta suprindo as necessidades, sem que $o$ interno tenha de sair.

De acordo com o protocolo, todos os profissionais em $\mathrm{SO}$ devem se paramentar com o uso da máscara N95 ou PFF2, touca, protetores para calçados, luvas de procedimentos, aventais impermeáveis por cima da roupa privativa (a ser trocada ao final do procedimento), além da face shield. Após a desparamentação, todos os profissionais são encaminhados para o vestiário, onde tomam banho e trocam a roupa privativa.

Por fim, o paciente realiza a recuperação da anestesia na própria $\mathrm{SO}$, para posterior encaminhamento diretamente para seu leito na unidade de internação (UI).

\section{DISCUSSÃO}

A pandemia da COVID-19 mudou a rotina dos estabelecimentos de saúde do mundo. Práticas anteriormente não consideradas passaram a ter status de essenciais para a prevenção de contaminação profissional e da contaminação cruzada $^{19}$. As taxas de utilização de SO diminuíram, aumentou o tempo de espera e também a notificação de incidentes, tanto no serviço da instituição deste estudo quanto em outros ${ }^{10,11}$.

O departamento cirúrgico organizou um comitê para a crise que integrou o comitê geral do hospital, como ocorreu em outros serviços ${ }^{2}$. Essa organização favoreceu o ciclo rápido de PDCA e adaptações quase que on-line com as novas diretrizes e com as experiências próprias a cada cirurgia.

O sucesso do enfrentamento da pandemia nos CC está relacionado, principalmente, à capacidade de previsão pelos gestores e profissionais para que sejam realizados, o mais precocemente possível, os preparativos e as adaptações com os devidos treinamentos, como neste relato ${ }^{9-11,20}$. Um dos fatores favoráveis ao sucesso da experiência relatada está no fato de o departamento cirúrgico não atender cirurgias de emergência, nas quais o risco de contaminação e a quebra do protocolo são ainda maiores ${ }^{10,11,20}$. Também cabe ressaltar o papel do protocolo anterior de checklist de cirurgia segura, visto que o momento da realização do checklist serviu, ao mesmo tempo, para a revisão dos pontos desse protocolo específico.

Os guidelines das principais sociedades do mundo utilizaram-se de evidências prévias para uma extrapolação que possibilitasse respostas rápidas para o preparo dos departamentos cirúrgicos, convergindo quanto às orientações ${ }^{16,18-21}$. Rotinas para pacientes em isolamento respiratório e de contato já eram amplamente difundidas nos CC, contudo, para o atendimento à COVID-19, os pacientes e os procedimentos geradores de aerossóis tomaram ainda mais relevância. Entretanto, o principal foco das publicações no primeiro semestre de pandemia foi como lidar com o cancelamento das cirurgias eletivas, como priorizar as que não deviam ser suspensas e como manejar os pacientes em espera ${ }^{7,10,11,21}$.

Uma das preocupações sobre a realização de cirurgias para pacientes portadores de COVID-19 é quanto à fumaça cirúrgica, produzida principalmente quando o eletrocautério é utilizado, e vem sendo estudada há mais de $40 \operatorname{anos}^{22}$. Alguns autores chegaram a sugerir que se evitasse o uso do eletrocautério e reforçaram que o bisturi bipolar gera menos fumaça que o monopolar ${ }^{13,23,24}$.

Um dos desafios foi a redução de profissionais em SO; contudo, com o envolvimento das equipes e a conscientização da universidade, foi possível manter alunos de graduação fora desses procedimentos, em outras atividades ambulatoriais e em enfermarias, assim como ocorreu em outros serviços ${ }^{7,10,11,24}$.

A implantação de um protocolo baseado em evidências trouxe para a equipe, além de mais segurança, maior sentimento de confiança para os profissionais ao realizar suas atividades. A mudança do clima institucional é notória quanto à promoção de unidade e parceria, em que os profissionais se auxiliam mutuamente, ainda mais na paramentação e na desparamentação, bem como em todo o trabalho. Várias referências reportam que, para a ocorrência de cirurgias eletivas no período da pandemia, o preparo da equipe e a disponibilidade de EPI são o estritamente necessário ${ }^{11,19,24,25}$. Além disso, o treinamento adequado contribui para impedir a disseminação do vírus do departamento cirúrgico para todo o hospital ${ }^{24}$.

No serviço, as cirurgias eletivas vêm sendo realizadas apenas conforme uma priorização própria de cada especialidade, como em outros locais, com considerações gerais quanto ao status pré-operatório da doença de base, das condições clínicas, da presença ou não de suspeita ou confirmação de contaminação pelo coronavírus ${ }^{7,10,11,21,26}$. A próxima etapa é a reabertura das atividades para todas as cirurgias eletivas, aguardando as orientações nacionais e internacionais e 
seguindo referências seguras para a priorização de casos e a reorganização da fila de procedimentos ${ }^{7,8,10,11}$.

A realização do protocolo de montagem e desmontagem de SO, com oclusão dos equipamentos com filme plástico e todas as adaptações, além de paramentação e desparamentação, aumentaram o tempo de uso de sala em aproximadamente uma hora, sem contar o tempo de recuperação pós-anestésica ${ }^{19}$.

Por fim, resta refletir sobre aspectos do protocolo elaborado que não foram passíveis de intervenção. As limitações estiveram principalmente na questão estrutural, visto que as SO possuíam sistema de exaustão convencional, sem pressão negativa ou filtro high-efficiency particulate air filter (HEPA $)^{19}$. Além disso, não foi possível abranger toda a equipe porque alguns se afastaram do serviço precocemente. Por isso, um profissional do time de treinamento permaneceu supervisionando as etapas de paramentação e desparamentação para todos os profissionais ${ }^{19}$. Outra limitação estrutural está no fato de que no departamento cirúrgico ainda são utilizadas ferramentas impressas para registros de sala, que, mesmo permanecendo fora da SO, tinham elevado risco de ser fômites para a disseminação do vírus SARS-CoV-2 ${ }^{19}$.

\section{CONCLUSÃO}

A elaboração e a implementação do protocolo de atendimento a pacientes suspeitos e confirmados de COVID-19 foram realizadas por um time de profissionais norteados por diretrizes nacionais e internacionais, treinamentos e atualizações. Os ciclos PDCA permitiram que o protocolo fosse aprimorado ao longo dos dois meses relatados. Barreiras e facilitadores para o processo foram identificados e estratégias foram elaboradas para adequações possíveis. Espera-se que muitos ganhos em termos de protocolos e procedimentos permaneçam para serem utilizados após a pandemia.

\section{REFERÊNCIAS}

1. Oliveira AC, Lucas TC, Iquiapaza RA. 0 que a pandemia da COVID19 tem nos ensinado sobre adoção de medidas de precaução? Texto Contexto Enferm. 2020;29:e20200106. https://doi. org/10.1590/1980-265x-tce-2020-0106

2. Peloso A, Moeckli B, Oldani G, Triponez F, Toso C. Response of a European surgical department to the COVID-19 crisis. Swiss Med Wkly. 2020;150:w20241. https://doi.org/10.4414/smw.2020.20241

3. The Lancet. COVID-19: protecting health-care workers. The Lancet. 2020;395(10228):922. https://doi.org/10.1016/S0140-6736(20)30644-9

4. Hussain K, Dewan V, Ali T, Al Shakarchi J. The impact of the COVID19 pandemic on the provision of surgical care. J Surg Case Rep. 2020;2020(4):rjaa087. https://doi.org/10.1093/jscr/rjaa087

5. Satomi E, Souza PMR, Thomé BC, Reingenheim C, Werebe E, Troster EJ, et al. Alocação justa de recursos de saúde escassos diante da pandemia de COVID-19: considerações éticas. Einstein (São Paulo). 2020;18:eAE5775. https://doi.org/10.31744/ einstein_journal/2020ae5775

6. Medeiros EAS. A luta dos profissionais de saúde no enfrentamento da COVID-19. Acta Paul Enferm. 2020;33:e-EDT20200003. https:// doi.org/10.37689/acta-ape/2020edt0003

7. Al-Jabir A, Kerwan A, Nicola M, Alsafi Z, Khan M, Sohrabi C, et al. Impact of the Coronavirus (COVID-19) pandemic on surgical practice - Part 1. Int J Surg. 2020;79:168-79. https://doi.org/10.1016/j. ijsu.2020.05.022
8. Covid Surg Collaborative. Elective surgery cancellations due to the COVID-19 pandemic: global predictive modelling to inform surgical recovery plans. Brit J Surg. 2020;107(11):1440-9. https://doi. org/10.1002/bjs.11746

9. Gilat R, Haunschild ED, Tauro T, Cole BJ. Recommendation to optimize safety of elective surgical care while limiting the spread of COVID-19: primum non nocere. Arthrosc Sports Med Rehabil. 2020;2(3):e177-e183. https://doi.org/10.1016/j.asmr.2020.04.008

10. Low TY, Hartman M, Chee CYJ, Mohankumar B, Ang SBL, San MT, et al. Restructuring the surgical service during the COVID-19 pandemic: experience from a tertiary institution in Singapore. Am J Surg. 2020:220(3):553-5. https://doi.org/10.1016/j. amjsurg.2020.05.021

11. Mariani NM, Pisani Ceretti A, Fedele V, Barabino M, Nicastro V, Giovenzana M, et al. Surgical strategy during the COVID-19 pandemic in a University Metropolitan Hospital in Milan, Italy. W J Surg. 2020;44:2471-6. https://doi.org/10.1007/s00268-020-05595-y

12. Rana RE, Ather MH, Enam SA. Change in surgical practice amidst COVID 19: example from a tertiary care centre in Pakistan. Ann Med Surg (London). 2020;54:79-81. https://doi.org/10.1016/j. amsu.2020.04.035

13. American College of Surgeons (ACS). COVID-19 and surgery. COVID19: elective case triage guidelines for surgical care [Internet]. ACS; 2020 [acessado em $1{ }^{\circ}$ dez. 2020]. Disponível em: https://www.facs. org/covid-19/clinical-guidance/elective-case 
14. Brat GA, Hersey S, Chhabra K, Gupta A, Scott J. Protecting surgical teams during the COVID-19 outbreak: a narrative review and clinical considerations. An Sug. 2020;10.1097/SLA.0000000000003926. https://doi.org/10.1097/SLA.0000000000003926

15. Center for Diseases Control and Prevention (CDC). Interim U.S. guidance for risk assessment and public health management of healthcare personnel with potential exposure in a healthcare setting to patients with coronavirus disease (COVID-19) [Internet]. CDC; 2020 [acessado em $1{ }^{\circ} \mathrm{dez} .2020$ ]. Disponível em: https://www.cdc.gov/ coronavirus/2019-ncov/hcp/guidance-risk-assesment-hcp.html

16. American College of Surgeons (ACS). COVID-19: considerations for optimum surgeon protection before, during, and after operation [Internet]. ACS; 2020. [acessado em 15 dez. 2020]. Disponível em: https://www.facs.org/-/media/files/covid19/considerations_optimum_ surgeon_protection.ashx

17. Forrester JD, Nassar AK, Maggio PM, Hawn MT. Precautions for operating room team members during the COVID-19 pandemic. J Am Coll Surg. 2020;230(6):1098-101. https://doi.org/10.1016/j. jamcollsurg.2020.03.03

18. Wick EC, Pierce L, Conte MC, Sosa JA. Operationalizing the operating room: ensuring appropriate surgical care in the era of COVID19. An Surg. 2020;272(2):e165-e167. https://doi.org/10.1097/ sla. 0000000000004003

19. Heffernan DS, Evans HL, Huston JM, Claridge JA, Blake DP, May AK, et al. Surgical Infection Society Guidance for Operative and Peri-Operative Care of Adult Patients Infected by the Severe Acute Respiratory Syndrome Coronavirus-2 (SARS-CoV-2). Surg Infec (Larchmt). 2020;21(4):301-8. https://doi.org/10.1089/sur.2020.101
20. COVID Surg Collaborative. Global guidance for surgical care during the COVID-19 pandemic. Brit J Surg. 2020;107(9):1097-103. https:// doi.org/10.1002/bjs.11646

21. Moletta L, Pierobon ES, Capovilla G, Constantini M, Salvador R, Merigliano S, et al. International Guidelines and Recommendations for Surgery During Covid-19 Pandemic: a systematic review. Int J Surg. 2020;79:180-8. https://doi.org/10.1016/j.ijsu.2020.05.061

22. Vourtzoumis P, Alkhamesi N, Elnahas A, Hawel JE, Schlachta C. Operating during COVID-19: is there a risk of viral transmission from surgical smoke during surgery? Can J Surg. 2020;63(3):E299-E301. https://doi.org/10.1503/cjs.007020

23. Hojaij FC, Chinelatto LA, Boog GHP, Kasmirski JA, Lopes JVZ, Sacramento FM. Surgical practice in the current COVID-19 pandemic: a rapid systematic review. Clinics (Sao Paulo). 2020;75:e1923. https:// doi.org/10.6061/clinics/2020/e1923

24. Spolverato G, Capelli G, Restivo A, Bao QC, Pucciarelli S, Pawlik TM, et al. The management of surgical patients during the coronavirus dieases 2019 (COVID-19) pandemic. Surgery. 2020;168(1):4-10. https://doi.org/10.1016/j.surg.2020.04.036

25. Goh SSN, Yeo DXW, Kaushal SA, Yeong HK, Tan GWL. Surgical and endoscopy protocols for general surgeons during the COVID19 pandemic: an institutional experience in Singapore. $\mathrm{Br} \mathrm{J}$ Surg. 2020;107(8):e249. https://doi.org/10.1002/bjs.11696

26. Presl J, Weitzendorfer M, Varga M, Borhanian K, Ciftci S, Emmanuel $\mathrm{H}$, et al. Surgical strategies during the COVID-19 crisis: the Salzburg concept. Am J Surg. 2020;220(3):550-2. https://doi.org/10.1016/j. amjsurg.2020.05.006 